\title{
Enrichment and Isolation of Biosurfactant Producers from Marine Environment
}

\author{
Shilpa Sabnis* and Vishal Juvale \\ Department of Microbiology, The Institute of Science, Mumbai, India \\ *Corresponding author
}

\section{A B S T R A C T}

Keywords

Marine, Biosurfactant producers, Surface tension reduction, Emulsification, Myroides spp.

\section{Article Info}

Accepted:

24 March 2016

Available Online:

10 April 2016
Biosurfactants are amphiphilic compounds produced by micro-organisms which reduce surface tension at the air-water interface facilitating properties like emulsification, foaming, detergency and dispersing. In the present study, seven bacterial strains isolated from 3 different marine environments were screened for biosurfactant production on the basis of Emulsification index (E24) Assay, Drop-collapse test, Oil spreading technique, CTAB Assay, Blood haemolysis test and Capillary Rise Method. Three biosurfactant producers showing maximal surface tension reducing potential were further identified on the basis of their biochemical characterization and 16S rRNA sequencing and found to be Myroides odoratimimus SKS05GRD, Myroides odoratimimus CM9 and Myroides odoratimimus SH9.

\section{Introduction}

Microorganisms play an important role in the maintenance and sustainability of any ecosystem as they are more capable of rapid adjustment towards environmental changes and deterioration. Bacteria are widespread in nature as they can adapt to any extreme environmental conditions and perform various physiological activities. Marine environments are one of the most adverse environments owing to their varying nature of temperature, $\mathrm{pH}$, salinity, sea surface temperature, currents, precipitation regimes and wind patterns. Due to the constant variation of environmental conditions, the microorganisms present in that environment are more suitably adapted to the adverse conditions, hence, possessing complex characteristic features of adaptation (Dash et al., 2012). Wealthy flora from marine biosphere represents a vast natural resource of imperative functional commercial grade products. Among the various bioactive compounds, biosurfactants are attracting major interest and attention due to their structural and functional diversity. Therefore, the bacteria isolated from the marine environments are supposed to be better utilized in biosurfactant production. Many marine bacteria have been reported to have potential to produce biosurfactants. The advantage of using marine bacteria for this is the direct use of organisms in any 
adverse conditions without any genetic manipulation (Satpute et al., 2010). Biosurfactants comprise a wide range of chemical structures, such as glycolipids, lipopeptides, polysaccharide-protein complexes, phospholipids, fatty acids and neutral lipids.

Moreover, these molecules can be tailormade to suit different applications by changing the growth substrate or growth conditions. Although most biosurfactants are considered to be secondary metabolites, some may play essential roles for the survival of biosurfactant-producing microorganisms through facilitating nutrient transport or microbe-host interactions or by acting as biocide agents. Biosurfactant roles include increasing the surface area and bioavailability of hydrophobic waterinsoluble substrates, heavy metal binding, bacterial pathogenesis, quorum sensing and biofilm formation. Biosurfactants are amphipathic molecules with both hydrophilic and hydrophobic moieties that partition preferentially at the interface between fluid phases that have different degrees of polarity and hydrogen bonding, such as oil and water or air and water interfaces. This property explains their broad use in environmental applications. Most work on biosurfactant applications has been focused on their use in environmental applications owing to their diversity, environmentally friendly nature, suitability for large-scale production and selectivity. Despite their potential and biological origin only a few studies have been carried out on applications related to the biomedical field. Some biosurfactants are suitable alternatives to synthetic medicines and antimicrobial agents and may be used as safe and effective therapeutic agents (Rodrigues et al., 2006).

Therefore, in the present study an attempt was made to enrich, isolate and identify biosurfactant producers from primarily three different oil-spilled marine environments. Once isolated, they can be further tested for various biomedical applications.

\section{Materials and Methods}

\section{Enrichment and Isolation of Biosurfactant Producers}

Oil-spilled seawater samples were collected from Vashi, Mandangad, \& Dapoli coastal region and inoculated into $100 \mathrm{ml}$ of St. Mineral salt medium containing $1 \%$ diesel oil as the sole carbon source. The flask was incubated at RT for 1 week on shaker at 150 rpm. It was serially enriched using $2 \%, 2.5 \%$ and 3\% diesel oil respectively in St. Mineral salt medium under same incubation conditions. A loopful of final enrichment broth was isolated on St. Mineral Salt Agar plates with $1 \%$ diesel oil as the only carbon source and incubated at RT for 3- 4 days. The isolates were Gram stained and preserved on St. Nutrient agar at $4^{0} \mathrm{C}$.

\section{Screening Assays for Detection of Biosurfactant Production}

\section{Drop-collapse Test}

The isolates were inoculated in St. Mineral salt medium with $1 \%$ diesel oil and incubated for $48 \mathrm{~h}$ and then centrifuged at $3000 \mathrm{rpm} / 15 \mathrm{mins}$ at $25^{\circ} \mathrm{C}$ to remove the cells. $2 \mu 1$ of diesel oil was applied to the well regions of a cavity slide and left to equilibrate for $24 \mathrm{~h}$. Five microlitres of the supernatant obtained after centrifugation was transferred to the oil-coated well regions on the cavity slide and the drop size was observed after $1 \mathrm{~min}$. Distilled water was used in place of the culture supernatant as the negative control.

\section{Oil spreading Technique}

Sudan red in liquid paraffin was added to the 
centre of a petriplate containing distilled water in a ratio of 1:8. The culture supernatant was now added to the petriplate at the centre. The biosurfactant producing organism was screened for on the basis of its ability to displace the oil and spread in the water.

\section{Blood Haemolysis Test}

The isolated cultures were streaked on sterile Blood Agar plates and incubated at $37^{\circ} \mathrm{C} / 48$ to $72 \mathrm{~h}$. Presence of a clear zone around the colonies was considered as positive.

\section{CTAB Assay or Blue Agar Plate Method}

A loopful of cultures spot inoculated on St. Mineral salt medium supplemented with glucose as carbon source (2\%) and cetyltrimethyl ammonium bromide (CTAB: $0.5 \mathrm{mg} / \mathrm{mL}$ ) and methylene blue (MB: 0.2 $\mathrm{mg} / \mathrm{mL}$ ) were used for the detection of anionic biosurfactant. Wells (4 mm diameter) were bored in the plate using a sterile cork borer. The cell free supernatant was loaded into the each well. The plate was then incubated at $37^{\circ} \mathrm{C} / 48$ to $72 \mathrm{~h}$. Presence of a dark blue halo around the well was considered positive for anionic biosurfactant production.

\section{Emulsification Assay}

The isolate cultures were centrifuged at $10,000 \mathrm{rpm} / 15 \mathrm{~min}$ at room temperature. The supernatant was then mixed with diesel oil and mixed vigorously for $2 \mathrm{~min}$. This mixture was left undisturbed for one hour at R.T. to separate aqueous phase from the oil phase. The absorbance of aqueous phase was measured colorimetrically at wavelength of $400 \mathrm{~nm}$. For determining the Emulsification index $\mathrm{E}_{24}$ of the isolates, the mixture of the culture supernatant with oil, after thorough mixing, was kept undisturbed for $24 \mathrm{~h}$. The Emulsification activity $\mathrm{E}_{24}(\%)$ was determined using following equation:

$$
\mathrm{E}_{24}(\%)=\frac{\text { The height of emulsion layer }}{\text { The height of total solution }} \times 100
$$

\section{Bacterial Adhesion to Hydrocarbons (BATH)}

Bacterial cells were washed twice with phosphate buffer salt solution $(\mathrm{pH} \mathrm{7)}$ and were suspended in it to give an optical density of $\sim 0.5$ at a wavelength of $600 \mathrm{~nm}$. Diesel oil was added to the cell suspension and vortexed for $3 \mathrm{~min}$. After shaking, the oil and aqueous phase were allowed to separate for $1 \mathrm{~h}$. The absorbance of the aqueous phase was then measured colorimetrically at a wavelength of $600 \mathrm{~nm}$. Hydrophobicity is expressed as the percentage of cell adherence to crude oil and was calculated as per the following formula:

Hydrophobicity $=(1-$ O.D. of aqueous phase / O.D. of initial cell suspension) $\times 100$

\section{Surface Tension Reduction}

Isolates were grown in MSM broth with $1 \%$ diesel oil and incubated at $120 \mathrm{rpm} / 7$ days. For surface tension measurements, $5 \mathrm{ml}$ of broth supernatant were transferred to a glass tube that was submerged in a water bath at a constant temperature $\left(28^{\circ} \mathrm{C}\right)$. Surface tension (S.T.) was calculated by measuring the height reached by the liquid when freely ascended trough a capillary tube. Noninoculated broth was used as control and the surface tension was calculated according to the following formula:

$$
\begin{gathered}
\gamma=\frac{r h \rho g}{2} \\
g=\text { Surface tension }(\mathrm{mN} / \mathrm{m}) ;
\end{gathered}
$$


$\mathrm{d}=$ Density $(\mathrm{g} / \mathrm{mL})$;

$\mathrm{g}=$ gravity $(980 \mathrm{~cm} / \mathrm{s} 2)$;

$\mathrm{r}=$ capillary radius $(0.05 \mathrm{~cm})$;

$\mathrm{h}=$ height of the liquid column $(\mathrm{cm})$.

The percentage Surface Tension Reduction was calculated by using the following formula:

$\%$ S.T. Reduction $=\mathrm{S} . \mathrm{T}$. of non-inoculated broth - S.T. of inoculated broth $\times 100$

S.T. of non-inoculated broth

\section{Identification of the Isolates}

The selected isolates were then characterized on the basis of their Gram nature, morphology and biochemical characteristics on comparison with Bergey's Manual of Systematic Bacteriology. These isolates were further identified by $16 \mathrm{~S}$ rRNA sequence analysis. The universal 16S rRNA eubacterial primer (50-GAGTTTGATC CTGGCTCAG-30; 50-AGAAAGGAGG TGATCCAGCC-30) was used for the amplification of $16 \mathrm{~S}$ rRNA. The $16 \mathrm{~S}$ rRNA gene sequence obtained from the all the 3 isolates was compared with other bacterial sequences by using NCBI mega BLAST (http://blast.ncbi.nlm.nih.gov/Blast. cgi) for their pair wise identities (Kiran et al., 2010).

\section{Results and Discussion}

Large quantities of crude oil, hydrocarbons, petroleum oil products and halogenated compounds finds their way into the marine ecosystem through accidental spillage (Satpute et al., 2010). Marine microorganisms have developed unique metabolic and physiological capabilities to thrive in extreme habitats and produce novel metabolites which are not often present in microbes of terrestrial origin (Satpute et al., 2010). Therefore, this rich marine habitat provides a magnificent opportunity to discover newer compounds such as antibiotics, enzymes, vitamins, drugs, biosurfactant (BS), bioemulsifier (BE) and other valuable compounds of commercial importance (Austin, 1989; Romanenko et al., 2001; Satpute et al., 2010).

Therefore, in the present study, marine water samples were collected from three different marine environments i.e. Vashi, Mandangad and Dapoli which are prone to hydrocarbon contamination and enriched for obtaining biosurfactant producers. Three successive enrichments were given to the water samples with increasing concentration viz. $2 \%, 2.5 \%$ and $3 \%$ diesel oil respectively as the only source of carbon in Mineral Salt medium (MSM).Growth of hydrocarbon degrader was seen in enrichment broths as turbidity. Presence of microorganisms was confirmed by Gram staining of enrichment broth. As the enrichment progressed very little lubricant oil could be detected in the medium. Large amounts of microorganisms were obtained after fourth enrichment of the water samples. The biosurfactant producer was finally isolated from MSM Broth onto MSM agar plates containing $1 \%$ diesel oil on incubation at R.T for 4 days. Well isolated colonies showing mucoid consistency were obtained. Most of hydrocarbon degrading bacteria are known to produce emulsifying substances; many of them are associated with extracellular matrix which assists bacteria in adhesion (Baldi et $a l ., 1999)$ and thus intake of non-aqueous phase liquids. Hence mucous production was one of the important criteria while selecting a biosurfactant producer. Seven colonies showing mucoid consistency were selected. These isolates were maintained on Nutrient Agar at $4^{\circ} \mathrm{C}$ for further study.

As the serial enrichment progressed, very little diesel oil could be detected in the 
medium. Large amounts of microorganisms were obtained after fourth enrichment of the water samples. The biosurfactant producer was finally isolated from MSM Broth onto MSM agar plates containing $1 \%$ diesel oil on incubation at R.T for 4 days. Well isolated colonies showing mucoid consistency were obtained. Most of hydrocarbon degrading bacteria are known to produce emulsifying substances; many of them are associated with extracellular matrix which assists bacteria in adhesion (Baldi et al., 1999) and thus intake of non-aqueous phase liquids. Hence mucous production was one of the important criteria while selecting a biosurfactant producer. Seven colonies showing mucoid consistency were selected. These isolates were maintained on Nutrient Agar at $4^{\circ} \mathrm{C}$ for further study.

\section{Screening of Biosurfactant-producing Bacteria}

Biosurfactants are structurally a very diverse group of biomolecules, e.g., glycolipids, lipopeptides, lipoproteins, lipopolysaccharides or phospholipids. Therefore, most methods for a general screening of biosurfactant producing strains are based on the physical effects of surfactants. Alternatively, the ability of strains to interfere with hydrophobic interfaces can be explored. On the other hand, specific screening methods like the colorimetric CTAB agar assay are suitable only to a limited group of biosurfactants. The screening methods can give qualitative and/or quantitative results. For preliminary screening of isolates, qualitative methods are generally sufficient (Walter et al., 2010).

Results of screening for biosurfactantproducing bacteria were analyzed and the most potential bacteria capable of producing biosurfactant was selected on the basis of results obtained from all the screening methods used in this study.
Drop Collapse Method is based on the ability of surfactants to destabilize liquid droplets on an oily surface. In the presence of surfactant, the liquid droplet spreads over the hydrophobic surface due to the reduction of interfacial tension between the liquid droplet and the hydrophobic surface. Otherwise, the droplet remains beaded in the absence of surfactant due to the repellence of water molecules from the hydrophobic surface (Walter et al., 2010). The dropcollapsing ability of seven bacterial isolates was tested on diesel oil and was found to give the best qualitative indication for the presence of surfactant action.

Out of the total 7 isolates screened by the drop collapse method, 4 isolates viz. $\mathrm{V}_{1}, \mathrm{~V}_{2}$, $\mathrm{M}_{1} \mathrm{~A} \quad \& \quad \mathrm{M}_{2} \mathrm{~B}$ showed less activity as compared to $\mathrm{V}_{\mathrm{s}}, \mathrm{M}_{1} \mathrm{~B} \& \mathrm{M}_{2} \mathrm{~B}$ though they were all positive for hemolytic and BATH assays. The reason behind the less activity in drop collapse test and positive hemolytic results obtained with above 7 isolates might be that some bacterial cells act as biosurfactant themselves and have high cell hydrophobicity, but do not produce extracellular biosurfactants (Table 1).

Oil Spreading Technique is a reliable method to detect biosurfactant production by diverse microorganisms based on the ability of the biosurfactants present in the supernatant of isolate solutions capable of spreading the oil and producing a clear zone.This clearing zone on the oil surface correlates to surfactant activity, also called oil displacement activity (Walter et al., 2010). Oil spreading assay results were in corroboration with drop collapse assay results. Strains found with positive drop collapse results were positive for oil spreading assay also. These results confirmed the presence (for strains with positive results) and absence (for strains with negative results) of surface active 
compound (biosurfactant) in the cell free culture broth. Morikawa et al., 2000 reported that the area of oil displacement in oil spreading assay is directly proportional to the concentration of the biosurfactant in the solution. However, in this study there was no quantitative study conducted on biosurfactant concentration versus oil spreading activity, but a qualitative study to check the presence of biosurfactant in the cell free culture broth was in concurrence with these reports. As found in drop collapse assay, 4 isolates viz. $\mathrm{V}_{1}, \mathrm{~V}_{2}, \mathrm{M}_{1} \mathrm{~A} \& \mathrm{M}_{2} \mathrm{~B}$ showed least oil spreading activity while $V_{s}$, $M_{1} B$ \& $M_{2} B$ were positive for the oil spreading assay (Table 1). Results of oil spreading technique show that all the seven isolates showed high oil spreading activity. Similar results with drop collapse and oil spreading assay was reported earlier (Youssef et al., 2004).

Hemolytic Assay is another qualitative assay to determine biosurfactant producer which is based on their ability to cause hemolysis of red blood cells. This test is based on the fact that surfactants interact strongly with cellular membranes and proteins. All the seven isolates used in the present study were found to show hemolysis on Blood Agar Plates. Out of these, isolates Vs, $\quad M_{1} B \quad \& \quad M_{2} B$ showed maximum hemolytic activity (Table 1). The $\beta$ hemolysis pattern was indicated by the formation of clear zone around the bacterial colonies grown on the Blood Agar. Carrillo et al., 1996 found an association between hemolytic activity and surfactant production and they recommended the use of blood agar lysis as a primary method to screen biosurfactant production. None of the studies reported in the literature mention the possibility of biosurfactant production without a hemolytic activity. However, in some studies hemolytic assay excluded many good biosurfactant producers and in some reports strains with positive hemolytic activity were found negative for biosurfactant production. In addition, not all biosurfactants have hemolytic activity and compounds other than biosurfactants may cause hemolysis. Hence, in the present investigation BATH assay, drop collapse test, oil spreading assay, emulsification assay and surface tension measurements were included to confirm biosurfactant production.

CTAB (cetyltrimethylammonium bromide) agar plate method is actually a semi-quantitative assay for the detection of extracellular glycolipids or other anionic surfactants. The microbes of interest are cultivated on a light blue mineral salts agar plate containing the cationic surfactant cetyltrimethylammonium bromide and the basic dye methylene blue. If anionic surfactants are secreted by the microbes growing on the plate, they form a dark blue, insoluble ion pair with cetyltrimethylammonium bromide and methylene blue. Thus, productive colonies are surrounded by dark blue halos.

In the present study, the seven isolates were unable to produce dark blue halos around the colonies (Table 1). As all the other screening methods were positive for biosurfactant production, a negative result using CTAB Assay is indicative of the fact that the biosurfactants produced by these isolates are either not glycolipids or they do not belong to other anionic biosurfactant groups.

Emulsification Assay is an indirect method used to screen biosurfactant production. It was assumed that if the cell free culture broth used in this assay contains biosurfactant then it will emulsify the hydrocarbons present in the test solution (Walter et al., 2010). In this study, diesel oil 
was used as the hydrophobic substrate. Results observed in this study revealed positive emulsification activity for all the seven isolates. Out of all the isolates used in the study, three isolates viz. Vs (0.39), $\mathrm{M}_{1} \mathrm{~B}$ (0.43) \& $\mathrm{M}_{2} \mathrm{~B}$ (0.57) showed the highest emulsification activity (Table 1).

Emulsification Index $\left(\mathbf{E}_{24}\right)$ is based on the emulsification capacity of biosurfactants. $\mathrm{E}_{24}$ correlates to the surfactant concentration. Evaluating the emulsification capacity is a simple screening method suitable for a preliminary screening of biosurfactant producing microbes (Walter et $a l ., 2010)$. Out of the seven isolates used in the study, three isolates viz. Vs, $\mathrm{M}_{1} \mathrm{~B} \&$ $\mathrm{M}_{2} \mathrm{~B}$ shown maximum $\mathrm{E}_{24}$ i.e. $53.33 \%, 50 \%$ $\& 60 \%$ respectively (Table 1 ).

BATH Assay is used to determine the cell hydrophobicity. Cell adherence with hydrophobic compounds like diesel oil is considered as an indirect method to screen bacteria for biosurfactant production, because cells attach themselves with oil droplets by producing surface active compounds called biosurfactants. All the seven isolates used in the present study were found to be positive for the BATH assay, which is indicative the affinity of the bacterial cells towards hydrophobic substrate. Cell adherence found for above positive isolates with diesel oil was in the range of 15.60 to $45.20 \%$. Maximum cell attachment with diesel oil was found with $\mathrm{M}_{2} \mathrm{~B}(45.20 \%)$ followed by $\mathrm{M}_{1} \mathrm{~B}(34.50 \%)$ \& Vs (33.30\%) (Table 1). A positive correlation was found between hemolytic assay and BATH assay. Positive cell hydrophobicity was reported as an indication of biosurfactant production. Visualization of bacterial cells adhered to crude oil confirmed the affinity of cells towards crude oil droplets (Thavasi et al., 2011).
Surface Tension Reduction and its measurement have been used by many researchers to study the surface properties of the biosurfactant. The direct measurement of the interfacial or surface activity of the culture supernatant is the most straightforward preliminary screening method for biosurfactant producing microbes. This gives a strong indication on biosurfactant production. The interfacial or surface tension of a liquid can be measured by a variety of methods. However, there is a restriction in the range of measurement (Walter et al., 2010). In the present study using the capillary rise method for measurement of surface tension of cell free culture broths revealed that all the seven isolates were capable of bringing about a reduction in surface tension. The highest surface tension reduction was observed with 3 isolates namely Vs $(18.51 \%), \quad \mathrm{M}_{1} \mathrm{~B}$ (30.21\%) \& $\mathrm{M}_{2} \mathrm{~B}(33.99 \%)$ (Table 1).

In fact, a direct correlation was found between drop collapse, oil spreading and surface tension assays. Strains highly active in any one of these methods were active in other two methods. Similar direct correlation between drop collapse method and surface tension has been reported by Bodour and Miller-Maier, 1998 and direct correlation between drop collapse and oil spreading method has been reported by Youssef et al., 2004. The results of the present study are thus at par with the earlier reports and thus stand justified.

Out of the seven isolates used in the study the three best candidate isolates viz. Vs, $\mathrm{M}_{1} \mathrm{~B} \& \mathrm{M}_{2} \mathrm{~B}$ showing positive activity in drop collapse, oil spreading and surface tension assays were selected and used for further study. 


\section{Identification of the Isolate}

\section{Morphological and Cultural Characteristics}

All of these isolates were found to be aerobic, Gram negative, rod-shaped, nonmotile bacteria, in overnight culture on Luria Bertani (LB) medium at $37^{\circ} \mathrm{C}$. The shapes of the colonies were also examined on the plates. After incubation for $24 \mathrm{~h}$ at $37^{\circ} \mathrm{C}$ on LB agar medium, the strain colonies were Yellow-colored, small shiny convex, circular smooth edges. The morphological features were confirmed using a light microscope. Under microscopy, the morphology of the isolated strains was revealed as short rods cells which are 3.5-4 $\mu \mathrm{m}$ in diameter \& $0.2-0.3 \mu \mathrm{m}$ in width. The optimum growth conditions of the strains were also investigated. All the strains were found to grow optimally at $37^{\circ} \mathrm{C}$ and $\mathrm{pH} 7$ with agitation at $150-250 \mathrm{rpm}$.

\section{Biochemical Characteristics}

The physiological and biochemical characteristics of the isolated strains were studied. All the tested isolates had both oxidase and catalase activity. All the 3 isolates were utilizing glucose aerobically but not in fermentative condition but they unable to utilize sucrose and fructose (Table 2). Based on their morphological, cultural, physiological, and biochemical characteristics, all strains were found to belong to the genus Myroides.

\section{$16 S$ rRNA Sequence Similarity and Phylogenetic Analysis}

Molecular identification of the isolated strains was carried out based on 16S rRNA sequence analysis. The $1.4 \mathrm{~kb}$ sequences obtained from the isolates were aligned with all the presently available $16 \mathrm{~S}$ rRNA sequences in the GenBank database. As a result, a phylogenetic tree was constructed using the neighbor joining method. Phylogenetic analysis using the $16 \mathrm{~S}$ rRNA sequences indicated that all the bacterial isolates belonged to the genus Myroides. From the branching pattern of the tree, all the three isolates (Vs, M1b \& M2b) were found to be close relatives to Myroides Odoratimimus rather than Flavobacterium (Fig. 1).

Table.1 Assays for Screening Biosurfactant Producers

\begin{tabular}{|l|l|l|l|l|l|l|l|}
\hline \multirow{2}{*}{ Screening assays } & \multicolumn{7}{|l|}{ Isolates } \\
\cline { 2 - 9 } & V1 & V2 & Vs & M1A & M1B & M2A & M2B \\
\hline Drop-collapse test & + & + & ++ & + & ++ & + & ++ \\
\hline $\begin{array}{l}\text { Oil spreading } \\
\text { technique }\end{array}$ & + & + & + & + & + & + & + \\
\hline Blood haemolysis test & + & + & + & + & + & + & + \\
\hline CTAB assay & - & - & - & - & - & - & - \\
\hline Emulsification assay & 0.15 & 0.15 & 0.39 & 0.3 & 0.43 & 0.35 & 0.57 \\
\hline Emulsification index & $46.66 \%$ & $43.33 \%$ & $53.33 \%$ & $43.33 \%$ & $50 \%$ & $40 \%$ & $60 \%$ \\
\hline $\begin{array}{l}\text { Bacterial Adhesion To } \\
\text { Hydrocarbons } \\
\text { (BATH) }\end{array}$ & $26 \%$ & $16 \%$ & $33.30 \%$ & $26.70 \%$ & $34.50 \%$ & $15.60 \%$ & $45.20 \%$ \\
\hline $\begin{array}{l}\text { Surface tension } \\
\text { reduction }\end{array}$ & $13.64 \%$ & $10.44 \%$ & $18.51 \%$ & $14.70 \%$ & $30.21 \%$ & $6.81 \%$ & $33.99 \%$ \\
\hline
\end{tabular}


Table.2 Biochemical Characteristics of the Biosurfactant Producers

\begin{tabular}{|l|c|c|c|}
\hline \multirow{2}{*}{ Biochemical Characteristics } & \multicolumn{3}{|c|}{ Results } \\
\cline { 2 - 4 } & $\mathbf{V s}$ & $\mathbf{M}_{\mathbf{1}} \mathbf{B}$ & $\mathbf{M}_{2} \mathbf{B}$ \\
\hline Glucose oxidative & + & + & + \\
\hline Glucose fermentative & - & - & - \\
\hline Sucrose oxidative & - & - & - \\
\hline Sucrose fermentative & - & - & - \\
\hline Fructose oxidative & - & - & - \\
\hline Fructose fermentative & - & - & + \\
\hline Oxidase & + & + & + \\
\hline Gelatinase & + & + & + \\
\hline NO ${ }_{3}$ reduction & + & + & + \\
\hline Urease & + & + & + \\
\hline Lipase & + & + & - \\
\hline Motility & - & - & - \\
\hline Lysine decarboxylase & - & - & - \\
\hline Citrate & - & - & + \\
\hline Indole Production & + & + & + \\
\hline Voges-Proskauer & + & + & - \\
\hline Starch utilization & - & - & Alkaline slant and \\
\hline TSI & alkaline slant and & Alkaline slant and & acidic butt \\
\hline
\end{tabular}

(Key: + Positive test, - Negative test)

Fig.1 Phylogenetic Tree of the Three Isolates. 16s rRNA Gene Phylotypes and Loosely Related Sequences from EMBL Database. The Topology shown was Obtained with the Neighbour-joining Method

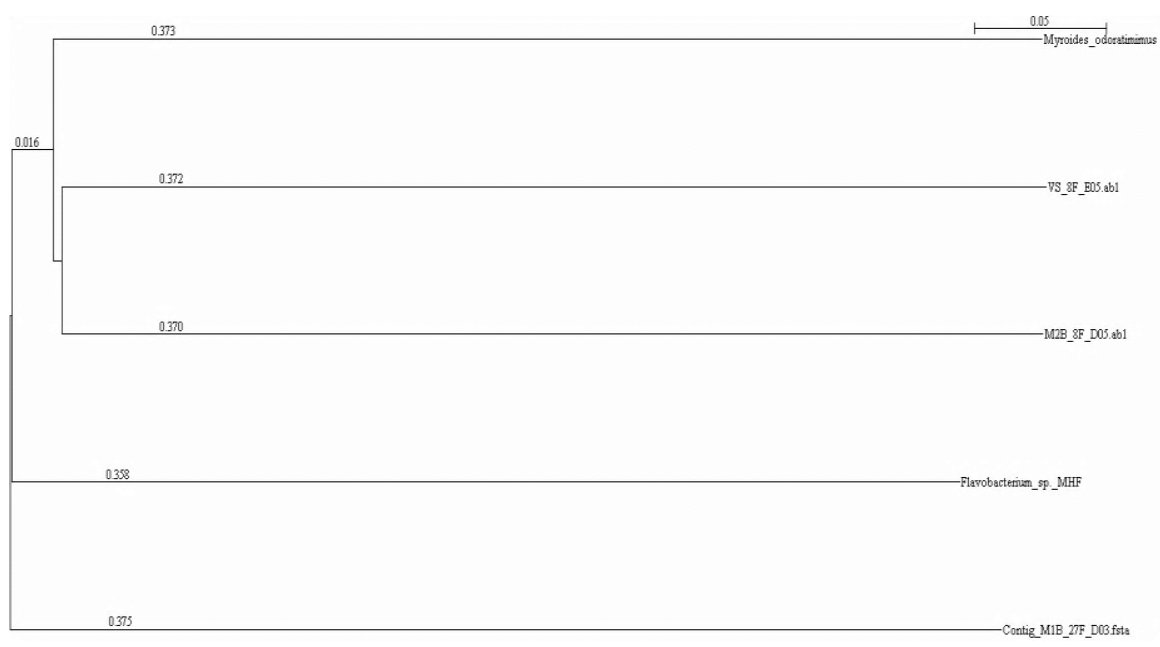

Therefore, the isolated strains were identified as Myroides species based on their morphological, cultural, physiological, and biochemical characteristics, as well as $16 \mathrm{~S}$ rRNA sequence analyses. Isolate Vs was identified to be M. odoratimimus SKS05-
GRD, isolate $\mathrm{M}_{1} \mathrm{~B}$ was identified to be $M$. odoratimimus $\mathrm{CM} 9$ \& isolate $\mathrm{M}_{2} \mathrm{~B}$ was was identified to be M. odoratimimus SH9.

Myroides spp are aerobic, gram-negative, non-motile, pigmented (yellow to-orange) 
rod shaped bacterium usually found in marine ecosystem. The majority of work carried out on bioemulsifier (BE) producing Myroides is found to be on strain sp. $\mathrm{SM}_{1}$ which was isolated from oil-spilled seawater in Songkhla Lake, Thailand. Myroides sp. $\mathrm{SM}_{1}$, grows well, emulsifying weathered crude oil and produce extracellular BE (complex of L-ornithine lipids-L-ornithine and a different couple of iso-3-hydroxy fatty acid and iso-fatty acid) that possesses high surface activity for oil displacement as compared with other surfactants. Bioemulsifiers from such an extreme environment shows higher stability at a broad temperature range. Cell associated surface active molecules from Myroides spp. possess high emulsification activity by adhering to weathered crude oil (Satpute et al., 2010).

With ever increasing reports regarding the therapeutic and biomedical properties of biosurfactants, these molecules will surpass the realm of surfactants and might emerge as highly valued molecules of therapeutic and biomedical importance in the near future.

\section{Acknowledgement}

The authors would like to sincerely express their gratitude towards the staff at 'The National Centre for Cell Sciences (NCCS), Pune, India' for helping them with the $16 \mathrm{~S}$ rRNA gene sequencing of their isolates.

\section{References}

Austin, B. 1989. Novel pharmaceutical compounds from marine bacteria. $J$. Pl. Bacteriol., 67: 461-470.

Bodour, A.A., Miller-Maier, R.M. 1998. Application of a modified dropcollapse technique for surfactant quantitation and screening of biosurfactant-producing microorganisms. J. Microbiol. Methods, 32: 273-280.

Dash, H.R., Das, S. 2012. Bioremediation of mercury and importance of mer genes. Int. Biodeterior Biodedegradation, 75: 207-213.

Franco Baldi, Nadica Ivošević , Andrea MinaccQAi, Milva Pepi, Renato Fani, Vesna Svetličić , Vera Žutić. 1999. Adhesion of Acinetobacter venetianus to Diesel Fuel Droplets Studied with In Situ Electrochemical and Molecular Probes. Appl. Environ. Microbiol., 65(5): 2041-2048.

Jensen, P.R., Fenical, W. 1994. Strategies for the discovery of secondary metabolites from marine bacteria; ecological perspectives. Ann. Rev. Microbiol., 48: 559-584.

Kiran, G., Thomas, A., Selvin, J., Sabarathnam, B., Lipton, A. 2010. Optimizatiom and characterization of a new lippopeptide biosurfactant produced by marine Brevibacterium areum MSA13 in solid state culture. Biores. Technol., 101: 2389-2396.

L1'gia Rodrigues, Ibrahim, M., Banat, Jose' Teixeira1, Rosa' rio Oliveira. 2006. Biosurfactants: potencial applications in medicine. J. Antimicrobial Chemother., 57: 609-618.

Morikawa, M., Hirata, Y., Imanaka, T. 2000. A study on the structurefunction relationship of the lipopeptide biosurfactants. Biochim Biophys Acta, 1488: 211-218.

Rengathavasi Thavasi, Shilpy Sharma, Singaram Jayalakshmi. 2011. Evaluation of Screening Methods for the Isolation of Biosurfactant Producing Marine Bacteria. J. Pet. Environ. Biotechnol., 2157-7463.

Romanenko, L.A., Kalinovskya, N.I., Mikhailov, V.V. 2001. Taxonomic composition and biological activity of microorganisms associated with a 
marine ascidian Halocynthia aurantium.Russian J. Marine Biol., 27: 291-295.

Surekha, K., Satpute, Ibrahim, M., Banat, Prashant, K., Dhakephalkar, Arun, G., Banpurkar, Balu, A., Chopade. 2010. Biosurfactants, bioemulsifiers and exopolysaccharides from marine microorganisms. Biotechnol. Adv., 28: 436-450.
Walter, V., Syldatk, C., Hausmann, R. 2010. Screening Concepts for the isolation of biosurfactant producing microorganisms. J. Biosci Tech., 1: 120-126.

Youssef, H., Duncana, K., Naglea, D., Savagea, K., Knappb, R., McInerney, M. 2004. Comparison of methods to detect biosurfactant production by diverse microorganisms. J. Microbiol. Methods, 56: 339-347.

\section{How to cite this article:}

Shilpa Sabnis and Vishal Juvale. 2016. Enrichment and Isolation of Biosurfactant Producers from Marine Environment. Int.J.Curr.Microbiol.App.Sci.5(4): 730-740. doi: http://dx.doi.org/10.20546/ijcmas.2016.504.084 